\title{
A Laboratory Demonstration of Coriolis Effects on Wind-Driven Ocean Currents
}

\author{
BY DAVID BEESLEY, JASON OLEJARZ,
}

AMIT TANDON, AND JOHN MARSHALL

\section{PURPOSE OF ACTIVITY}

Here we study the mechanism by which the wind drives ocean circulation. We induce cyclonic and anticyclonic circulation by blowing air over the surface of a rotating tank of water using fans co-rotating with the turntable, as sketched in Figure 1. The Ekman layers and patterns of upwelling and downwelling can be visualized by using dye crystals and paper dots.

The flow within the surface Ekman layer associated with the action of the wind is convergent in anticyclonic flow and divergent in cyclonic flow if the apparatus is rotating cyclonically ( $\Omega>0$, corresponding to the northern hemisphere), as sketched in Figure 2. The convergent flow drives downward vertical motion (called Ekman pumping, Figure 2a); the divergent flow drives upward vertical motion from beneath (called Ekman suction, Figure 2b).

The experiment demonstrates how divergence in Ekman transport leads to vertical motion. Students visualize how continuity of the flow leads to upwelling in a cyclone and downwelling in an anticyclone, which then helps make connections with gyre-scale ocean circulation. Students learn how to visualize circulation at the surface and at depth using paper dots and dye tracers. (a) anticyclone

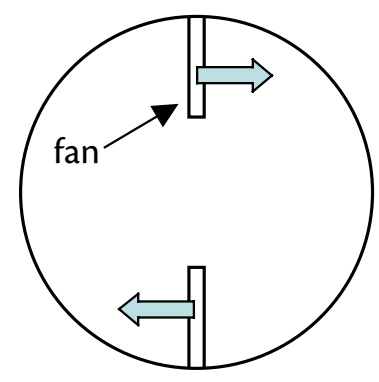

(b) cyclone

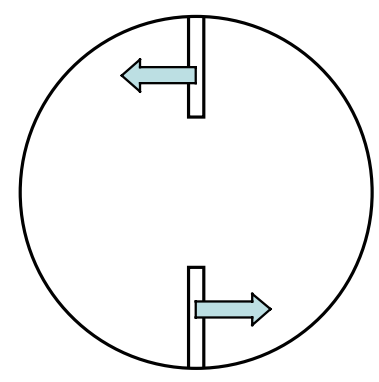

Figure 1. (a) Anticyclonic and (b) cyclonic circulation are induced by blowing air over the surface of a rotating tank of water using corotating fans in a study of how the wind drives ocean circulation. The arrows indicate the direction of the air flow in the rotating frame.
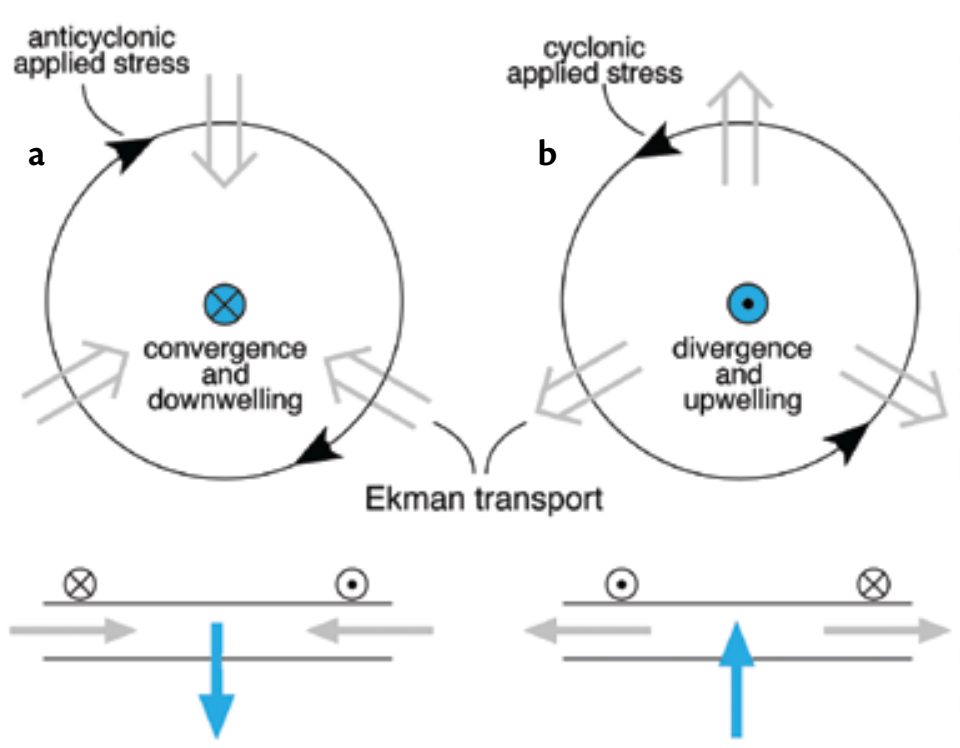

Figure 2. The Ekman transport is directed perpendicular to the applied stress (to the right if $\Omega>0$, to the left if $\Omega<0$ ), driving (a) convergent flow if the stress is anticyclonic and (b) divergent flow if the stress is cyclonic. (The case $\Omega>0$-appropriate to the northern hemisphere-is shown.) 


\section{AUDIENCE}

This demonstration is useful for marine science and meteorology undergraduates and graduate students as well as physics and engineering undergraduates and graduate students.

\section{BACKGROUND}

The deflecting nature of Coriolis forces on atmospheric and oceanic currents can be somewhat counterintuitive and difficult to visualize. ${ }^{1}$ The influence of the Coriolis force on ocean currents was first noted by the remarkable observations of Norwegian scientist and explorer Fridtjof Nansen (1861-1930). Nansen designed a vessel for the specific purpose of freezing it into Arctic ice and allowing it to drift with the ice over a year. In 1893, he positioned his 128-ft wooden ship, Fram, so that it would freeze into Arctic pack ice $1000 \mathrm{~km}$ south of the North Pole. Fram remained locked in the ice for 35 months. Nansen observed that the direction of ice and ship movement was not parallel to the wind but typically at an angle of $20^{\circ}-40^{\circ}$ to the right of it. Nansen related the problem to Vilhelm Bjerknes at the University of Uppsala, who assigned working out the theory to his student V. Wilfrid Ekman (1905). Wind-driven currents deflected by the Coriolis force are now known as Ekman currents.

Here we describe how computer cooling fans blowing air over water in a circular container placed on a rotating turntable can be used to represent the action of the wind on the ocean. Paper dots can be used to track the movement of the surface layers of the water. Thus, we are able to confirm Nansen's observations in the laboratory and also study the vertical circulations associated with wind-driven currents.

\section{MATERIALS}

This demonstration ${ }^{1}$ needs a turntable, a circular tank or a square tank with circular insert to remove the corner effects, water, two computer cooling fans, black paper dots to reveal the pattern of surface flow, and a few milligrams of potassium
David Beesley and Jason Olejarz are undergraduate physics seniors at the University of Massachusetts, Dartmouth, MA, USA. Amit Tandon (atandon@umassd.edu) ) is Associate Professor, Physics Department, University of Massachusetts, Dartmouth, MA, USA. John Marshall is Professor, Program in Atmospheres, Oceans, and Climate, Department of Earth, Atmospheric, and Planetary Sciences, MIT, and Director, MIT Climate Modeling Initiative, Cambridge, MA, USA.

permanganate crystals ${ }^{2}$ to display flow characteristics on the bottom of the tank. To create cyclonic and anticyclonic circulation, we gently blow air over the surface of the water using fans directed tangentially to the circular insert, as shown in Figure 1. Cooling fans used in personal computers are readily available in most electronic stores and work perfectly. The fans are driven by a $12 \mathrm{~V}$ DC power supply on the turntable, and their speed can be controlled via a potentiometer knob.

\section{ACTIVITY}

Before attaching the fans, decide whether you want to create a cyclone (fans circulate air in direction of rotation) or an anticyclone (fans circulate air opposite to the sense of rotation). The fans should then be positioned perpendicular to the wall of the tank at the point where the circular insert touches the square container, as shown in Figures 1, 3, and 4. The fans are clamped to the outer wall (green clamps in Figure 3). The fan must be held firmly and arranged to blow horizontally. Next, place the other fan on the opposite wall and direct it to blow in the reverse direction of the first fan. Figure 4 shows a view from the top.

Once the fans are mounted, fill the tank so that the water level is just below the bottom of the fans. Set the table rotating at about $10 \mathrm{rpm}$ (revolutions per minute). If the water starts to touch the bottom of the fans, remove small amounts of water until the water no longer touches. This helps reduce any waves

\footnotetext{
1 The "Weather in a Tank" (http://paoc.mit.edu/labguide) demonstrations are being implemented at a group of universities (University of Wisconsin, Madison; Penn State; Johns Hopkins University; Millersville University; and University of Massachusetts, Dartmouth, led by MIT) to introduce many atmospheric and oceanic-circulation-related concepts to students at various levels. A low-cost and easily transportable rotating table is used in a series of experiments that simulate diverse processes in the atmosphere-ocean system. The rotating table helps students understand the kinematics in a rotating frame of reference, and an extensive set of experiments has been designed for demonstrations. For more information about the apparatus required to carry out this and related experiments, contact John Marshall (jmarsh@mit.edu).

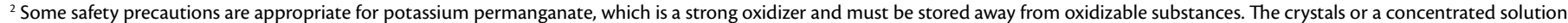
stains skin and clothing, but may be washed away using acetic acid. Brown epidermal skin stains disappear within a few days. The amount needed for this experiment is a few milligrams, a

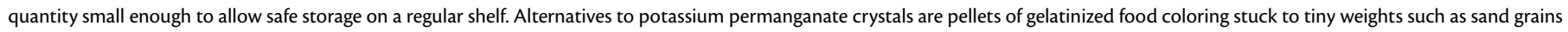
or crystals of colored sugar (available for decorating cakes).
} 
from the fans' vibrations. Let the water attain solid-body rotation for about 10 minutes before turning on the fans. During the spin-up phase, we typically discuss solid-body rotation and ask the students what they expect to see happen once the fans are turned on. The fans are run for another 10 minutes or so before adding paper dots and potassium permanganate crystals.

\section{Anticlockwise (Cyclonic) Case}

If the fans are mounted such that the wind stress generates an anticlockwise flow (known as cyclonic flow), the Ekman transport at the surface is directed outwards towards the walls, as sketched in Figure $2 \mathrm{~b}$. This transport is directly analogous to the ice drift Nansen observed in the Arctic and can be observed readily by sprinkling a few black paper dots on the surface. Over time (a few minutes), all of the dots floating on the sur-

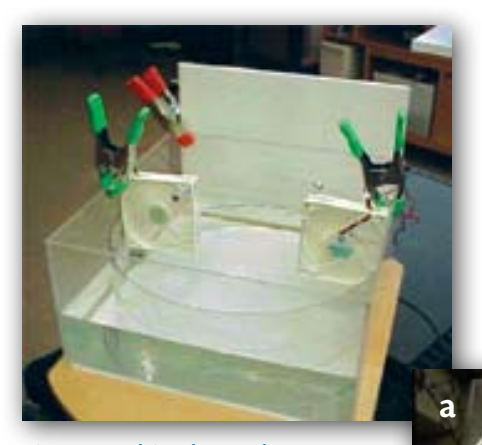

Figure 3. This photo shows the clear square tank sitting on a turntable. The computer fans are clamped to the sides. Also visible is an insert rendering a square tank circular. A white sheet acts as a back screen to visualize the flow in the vertical plane.
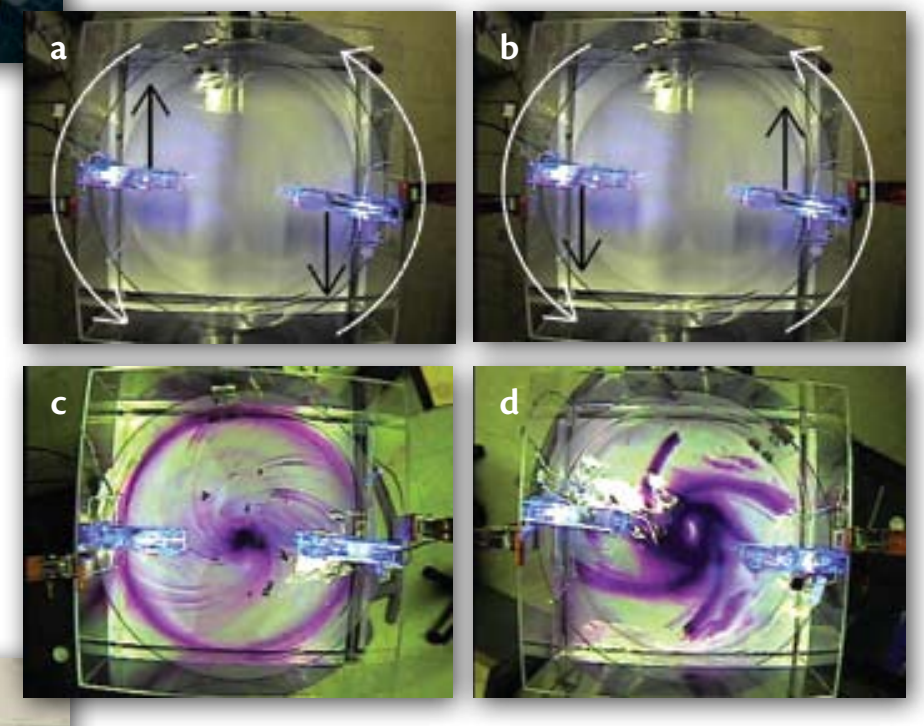

face make their way to the periphery. Such outward flow at the surface creates divergence. The fluid is thus drawn up from the interior. Flow at the bottom is convergent, which can be made easily visible by dropping in permanganate crystals, as seen in Figure $4 \mathrm{~d}$. The students can also observe the dye rising near the center (Figure 5).

We have found it most instructional to introduce the paper dots first, discuss their behavior, and only then mark the deep flow with permanganate crystals.

\section{Clockwise (Anticyclonic) Case}

If the fans are mounted such that the wind stress is clockwise (or anticylonic, in the jargon), they create Ekman convergence near the center of the tank at the surface, which forces fluid down, and creates an anticyclone near the bottom of the tank, as sketched in Figure 2a and seen in Figure 4c.

For best visual results, the floating black paper dots that are used as surface-flow tracers should be introduced near the center at the surface for the cyclone experiment, as their outward motion at the surface becomes quite clear. Likewise, for the anticyclone experiment, it is best to drop them near the edge of the tank. Potassium permanganate crystals are best dropped
Figure 4. Top row: (a) Anticyclonic and (b) cyclonic circulation are induced by blowing air over the surface of a rotating tank of water using co-rotating fans. In both cases, the table rotates anticlockwise (cyclonically) as shown by white arcs in the top row. The fan arrangements provide an anticyclonic wind stress in the left panel (a) and a cyclonic wind stress in the right panel (b). Black arrows show the direction of the air blown from the fans. Bottom Row: (c) Anticyclonic (left) and (d) cyclonic (right) circulations set up by the applied wind stress revealed by potassium permanganate crystals resting on the bottom of the tank. Black paper dots floating on the surface reveal the sense of the upper (directly wind-driven) flow. Over time, the dots congregate in the middle of the anticyclone but on the periphery of the cyclone. 
at the corners of an imaginary equilateral triangle centered in the middle of the tank and extending out to, roughly, half the radius of the tank.

The experiments are very robust and can be done with a variety of fan sizes and fan speeds, although slower speeds work best. We used fan sizes of 40,80, and $120 \mathrm{~mm}$ : we recommend using $120-\mathrm{mm}$ cooling fans rotating at the lowest speed. If the tank is filled and fans properly attached, it takes a 50-minute class period to do the demonstrations. In our implementation, we have used the preceding and following class periods to discuss the associated theory and implications.

\section{THEORY}

Theory tells us that the mass transport in an Ekman layer, $\mathbf{M}_{\mathrm{Ek}}$, is given by,

$$
\mathbf{M}_{\mathrm{Ek}}=\left(\boldsymbol{\tau}_{\text {wind }} \times \mathbf{z}\right) / \mathrm{f}
$$

where $\tau_{\text {wind }}$ is the stress applied by the wind at the surface, $\mathbf{z}$ is a unit vector pointing vertically upwards, $\mathrm{f}$ is the Coriolis parameter (in our laboratory experiment, $f=2 \Omega$ ), and “ $x$ ” is the vector product. We thus see that the mass transport of the Ekman layer is exactly to the right of the surface wind (in the northern hemisphere). The above equation determines $\mathbf{M}_{\mathrm{Ek}}$, which depends only on $\boldsymbol{\tau}_{\text {wind }}$ and $\mathrm{f}$. It does not predict typical velocities or boundary layer depths, which depend on the details of the turbulent boundary layer. A more complete analysis (carried out by Ekman, 1905) shows that the horizontal velocity vectors within the layer trace out a spiral, as shown in
Figure 6. But, irrespective of these details, the transport is to the right of the applied stress.

In our laboratory experiment, the Ekman layers (both at the top and the bottom) are very shallow, perhaps a few millimeters in depth. The majority of the fluid column is in geostrophic balance.

Figure 7 gives a broad view of how our laboratory experiment works. When the fans are arranged to create an anticyclonic (cyclonic) vortex, the ageostrophic flow at the surface is directed "to the right" of the wind direction and, hence, induces convergence (divergence) and downwelling (upwelling). The situation is reversed at the bottom of the tank. There, the stress opposes the direction of the large-scale interior flow, inducing ageostrophic flow at the bottom that is directed outward (inward) in an anticyclone (cyclone), as Figure 7 shows. The circulation sense sketched in Figure 7 is very evident in the patterns of permanganate streaks on the bottom observed in the experiment-see the bottom row of Figure 4.

\section{REAL-WORLD EXAMPLES}

As studied in detail by Ekman, when wind blows across the surface of the ocean, the momentum from the wind is transferred to the water via the wind stress on the surface. Earth's rotation then imparts a Coriolis acceleration on the moving water parcels that is perpendicular to the velocity and directed to the right in the northern hemisphere. Convergence in Ekman transport leads to downwelling, for example, in subtropical ocean gyres where low-latitude easterlies and mid-

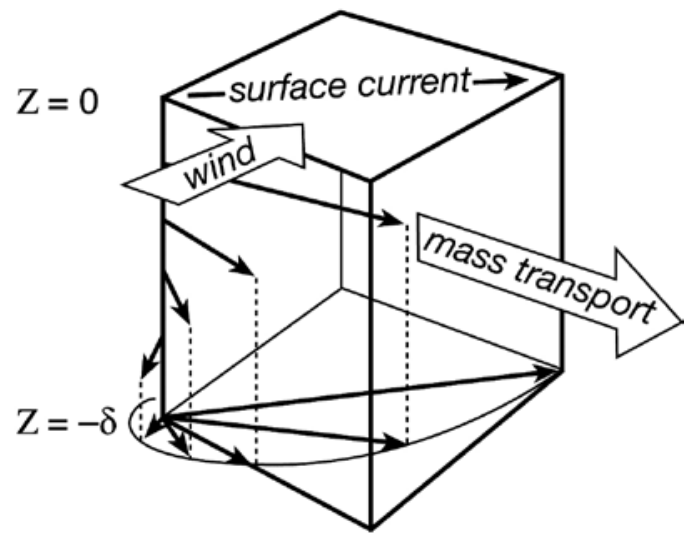

Figure 6. Mass transport of the Ekman layer is directed to the right of the wind in the northern hemisphere. Theory suggests that horizontal currents veer with depth as shown. This is called the Ekman spiral. (a) anticyclone
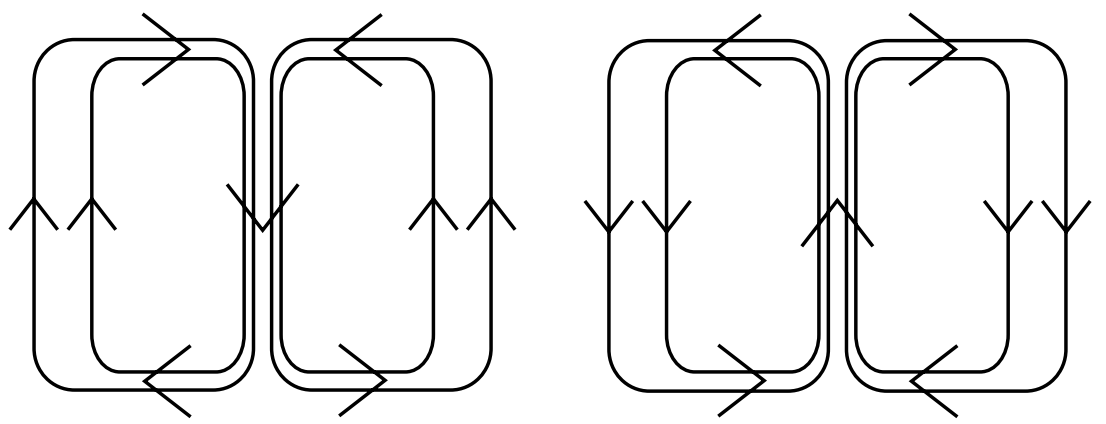

Figure 7. Schematic diagram showing ageostrophic vertical overturning circulation in an anticyclonic and in a cyclonic vortex. 


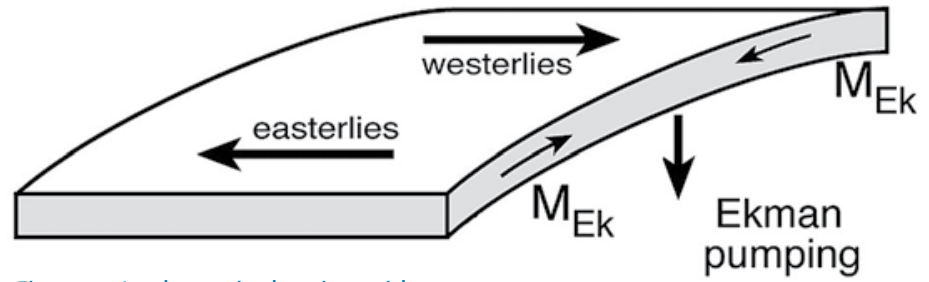

Figure 8. A schematic showing midlatitude westerlies (eastward wind stress) and tropical easterlies (westward stress) blowing over the ocean. Because the Ekman transport is "to the right of the wind" in the northern hemisphere, there is convergence and downward Ekman pumping into the interior of the ocean. Note that the sea surface is high in regions of convergence.
Figure 9. The global pattern of Ekman vertical velocity (in units of meters per year) fluxing through the base of the ocean's wind-driven surface layer. Motion is upward in the green areas, downward in the brown areas. Vertical motion is not computed over the white strip along the equator because of the Coriolis parameter, $f \rightarrow 0$, there. The thick line is the zero contour.

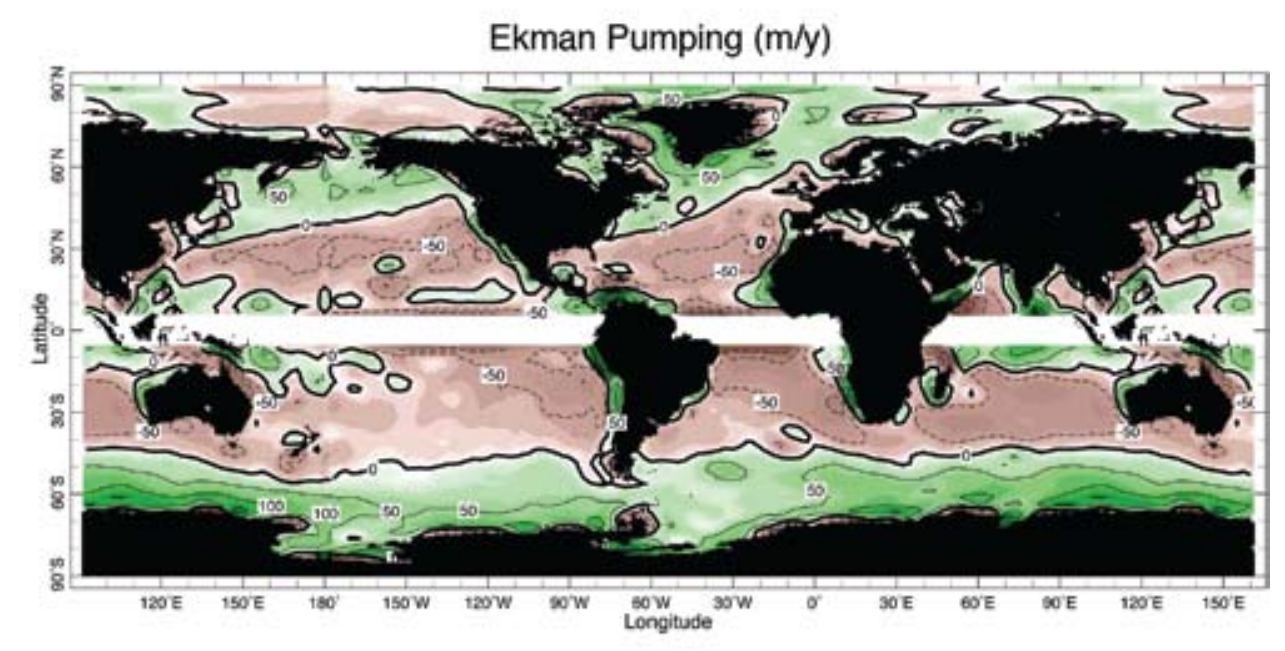

latitude westerlies force an Ekman convergence and hence lead to Ekman pumping (Figure 8). Divergence in Ekman transport leads to upwelling. For example, southerly winds on the east coast of a continent in the northern hemisphere lead to coastal upwelling. Likewise, subpolar ocean gyres, where the wind stress is a combination of westerlies to the south and polar easterlies in the north, result in upwelling (Ekman suction).

Figure 9 shows the global pattern of Ekman pumping/suction. The units are in meters per year $(\mathrm{m} / \mathrm{y})$. Typical downwelling/upwelling rates are $50 \mathrm{~m} / \mathrm{y}$. At this rate, it takes 20 years for water to move a distance of $1 \mathrm{~km}$ !

Finally, we mention one further, less-savory manifestation of Ekman layers. A vast area of plastic debris and other flotsam the size of the continental United States has been observed drifting in the top few tens of meters of the northern Pacific Ocean. ${ }^{3}$ It is held there by swirling wind-driven currents, just as are the paper dots floating on the surface of our laboratory experiment. The center of the vortex seems to be near Midway, an atoll (roughly $30^{\circ} \mathrm{N}, 180^{\circ} \mathrm{W}$ ) halfway between North America and Japan. Despite the fact that only a few dozen people live on the atoll, the seas surrounding it are full of debris-debris drawn in the from the periphery of the Pacific subtropical gyre by surface winds.

\section{ADDITIONAL ONLINE RESOURCES}

The Web sites mentioned below contain additional material on coastal and equatorial upwelling, as well as other rotating tank experiments.

- http://oceanmotion.org/html/background/upwelling-anddownwelling.htm

- http://paoc.mit.edu/labguide

\section{ACKNOWLEDGEMENTS}

We would like to acknowledge helpful comments by Jack Whitehead (Woods Hole Oceanographic Institution) on an earlier version of this article. This work is supported by the National Science Foundation via a CCLI Phase II award to John Marshall and Lodovica Illari at MIT. We thank Elsevier for granting permission to reproduce Figures 2, 6, 8, and 9 from Marshall and Plumb (2008), Chapter 10, Copyright Elsevier, 2008. 四

\section{REFERENCES}

Ekman, V.W. 1905. On the influence of the Earth's rotation on ocean currents. Arkiv for Matematik, Astronomi, och Fysik 2 (11)1-52.

Marshall, J., and R.A. Plumb. 2008. Atmosphere, Ocean and Climate Dynamics: An Introductory Text. Academic Press, 344 pp.

${ }^{3}$ See http://www.mindfully.org/Water/2006/Sea-Plague-Plastic2aug06.htm and The Independent (London) February 5, 2008. 